\title{
Light Transport Simulation and Realistic Rendering: State of the Art Report
}

\author{
Vladimir Frolov ${ }^{1,2}$, Alexey Voloboy ${ }^{1}$, Sergey Ershov ${ }^{1}$ and Vladimir Galaktionov ${ }^{1}$ \\ ${ }^{1}$ Keldysh Institute of Applied Mathematics RAS, Miusskaya sq. 4, Moscow, 125047, Russia \\ ${ }^{2}$ Moscow State University, Moscow, 119991, Russia
}

\begin{abstract}
The field of light transport simulation quickly growths in last decades. Nowadays there are about hundreds of books and papers that are quite difficult to cover for applied researcher or developer. Unlike similar surveys, in this paper we make attempt to provide short roadmap to select the best method for some light transport problem based on scene and calculated phenomena constraints. In our paper we propose several classifications for light transport simulation algorithms based on their mathematical properties, robustness and required scene constraints. These classifications help to understand advantages, disadvantages and limitations of the methods. In this paper we use not only a survey of existing works but also our own experience with the methods that we have implemented over the last decade in different software products. Some results of our experiments are shown in the paper. Finally, we propose a short guide for method selection in form of block scheme.
\end{abstract}

\section{Keywords}

Light transport simulation, Monte Carlo integration, realistic rendering, ray tracing.

\section{Introduction}

In order to classify light transport methods, we should understand the basic properties and characteristics by which we will make the classification. At the same time classification of methods allows as to build some kind of a road map of existing methods and propose a rule for appropriate method selection. Let's start with basic definitions of notions we operate in text below:

- $\quad$ OMC stands for Ordinary Monte Carlo integration.

- MCMC stands for Markov Chain Monte Carlo.

- Robustness. Let's consider lighting simulation algorithm is robust [1] if there are no outliers in the rendering equation calculation. In other words, there are not sparse Monte Carlo samples with extremely large values that prevent the convergence of the integral calculation in a reasonable time. More robust methods allow us to calculate more complicated illumination phenomena. This is why robustness is an extremely important characteristic.

- The convergence of the Monte Carlo method is inversely proportional to error. So if convergence increases then the error decreases. For example, if the convergence $C(N)=\sqrt{N}$ ( $N$ is the number of samples) then the error decreases proportionally to $\frac{1}{\sqrt{N}}$. Therefore in this case we have to increase the number of samples by a factor of 4 if we want to improve the accuracy in two times.

- The efficiency of the light transport simulation method is defined differently for OMC and MCMC methods.

GraphiCon 2021: 31st International Conference on Computer Graphics and Vision, September 27-30, 2021, Nizhny Novgorod, Russia EMAIL: vfrolov@graphics.cs.msu.ru (V. Frolov); voloboy@gin.keldysh.ru (A. Voloboy); ersh@gin.keldysh.ru (S. Ershov); vlgal@gin.keldysh.ru (V. Galaktionov) ORCID: 0000-0001-8829-9884 (V. Frolov); 0000-0003-1252-8294 (A. Voloboy); 0000-0002-5493-1076 (S. Ershov); 0000-0001-6460-7539 (V. Galaktionov)

(c) (i) 2021 Copyright for this paper by its authors.

Use permitted under Creative Commons License Attribution 4.0 International (CC BY 4.0).

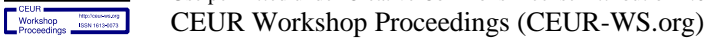


1. For the OMC methods this is the percentage of Monte Carlo samples that contribute significantly to the image. A contribution is significant if its luminance is comparable in orders of magnitude with the average luminance of the image or is significantly greater.

2. $\quad$ For the MCMC methods this is the average acceptance rate.

Recently we published extended paper [2] on this topic. Here we present the result of further topic elaboration. Current paper is to some extend another glance on the problem. But we recommend [2] for description of many details.

\section{Classification \#1: OMC vs MCMC}

From the beginning the light transport simulation was developed mainly in two ways on the basis of the Ordinary Monte Carlo (OMC) or the Markov Chain Monte Carlo (MCMC) [3]. Both groups of methods are successfully used now [4]. Of course, each group of methods has its advantages and disadvantages.

\subsection{OMC Branch}

All modern OMC-based light transport methods are based on Light tracing (LT), Path tracing (PT) and a Multiple importance sampling (MIS) technique [1]. MIS is a weighted sum of the contributions from several sampling strategies. Strategy can be explicit, like for example shadow rays, and implicit, for example, rays randomly hitting the light source. The main difficulty of this technique is the computation of sample weights.

Unfortunately the computation of MIS sample weights requires correct calculation of a Probability Density Function (PDF) for each sample. This significantly complicates the development of the material sampling (implicit strategies) and light sampling (explicit strategies). As a result only high level expert is able to do this [5].

The majority of commercial realistic rendering systems used in architecture, cinema and animation are based on the Path Tracing algorithm [4] using MIS (MIS PT). It is robust in calculation of the direct light. As soon as indirect (secondary) light becomes significant, more complex sampling strategies should be introduced. The method becomes complicated because its implementation has to work-around a lot of special cases like, for example, point and directed light sources, skylight, sky-portals, specular materials, BSDF composition etc.

The Bidirectional Path Tracing (BPT) is more complicated method [1]. Two paths are traced from the light source and from the camera with the interreflection depth $N$ and $M$ respectively. All complete paths from the source to the camera are formed using $N * M$ connections between the vertices of these paths. For each complete path its contribution is taken into account with the MIS weights. The Instant Bidirectional Path Tracing (IBPT) [6] and Probabilistic Connection BPT (PCBPT) [7] are further elaboration of the original BPT algorithm [1]. They are more efficient because they rarely use (PCBPT) or do not use at all (IBPT) strategies with intermediate connections. These strategies are often unsuccessful.

However, we can say that all three methods require that the models be symmetric. In fact, this requirement can be easy not met because asymmetrical input data [1]: the specific surface model [8], tracing of the polarized light usually is defined only in one direction $[9,10]$, and the specific media refractions [11] can cause asymmetry. The verification of bidirectional methods (especially BPT and PCBPT that use strategies with intermediate connections) is complicated because a lot of cases with different strategies which make a considerable contribution to the image must be considered.

The paper [12] proposed a sampling strategy if it is needed to simulate SDS (specular-diffusespecular) caustics within pure BPT. The most significant problem in the implementation of the method [12] is necessity to use differential geometry framework which directly affects the representation of geometric models within the system.

Apart of the methods mentioned above there are a lot of heuristic approaches that simulate certain individual phenomena and perform well for particular classes of scenes. They are described in [2]. However these approaches are not universal because the integration space is multidimensional and 
complex: in such a space each assumption made by a heuristic is sooner or later violated. The only method that ensures robustness in this case is the Multiple Importance Sampling (MIS). However its implementation for some approaches is very complicated. As a result different methods should be used in different cases which make light transport simulation problem extremely difficult for both developers and users of rendering systems.

\section{2. $\mathrm{MCMC}$ Branch}

The Markov Chain Monte Carlo (MCMC) is to some extend a generalization of the ordinary Monte Carlo method. The samples are correlated in the MCMC contrarily to the ordinary Monte Carlo where they are independent. So it is possible to reuse information in regions with high contribution. The Metropolis algorithm (or the Metropolis-Hastings algorithm) is the most popular version of the MCMC. The aim of all MCMC algorithms is to construct the distribution of samples proportionally to an arbitrary contribution function.

The Metropolis algorithm applied for the light transport problem is called Metropolis Light Transport $(M L T)$ [13]. It generates samples proportionally to the entire lighting integral rather than proportionally to a part of the integrand that is done by every BPT strategy. The MLT automatically places more samples into more significant parts of the integral function reducing the variance [14]. The issue of convergence for the MLT is more complicated. In particular, the estimate $O\left(\gamma^{N}\right)$, where $\gamma \in(0 ; 1)$, can be found in [15].

Note that the MIS and the Metropolis algorithm are not opposites. They can be used together and this is profitable. This was demonstrated in the first work on the MLT [13] where the Metropolis algorithm was proposed for the BPT and transitions in the Path Space were used as small changes of locations of the path vertex positions.

\subsubsection{Classification for MCMC methods}

The MCMC methods applied to rendering can be divided into two classes (Figure 1). The first class consists of the methods that work in the space obtained by the concatenation of all vertex coordinates in the world space. It is called the Path Space. Here the most valuable algorithms are Veach MLT [13], MEMLT [16] and HSLT [17]. The second class includes the methods that work in the space of all random numbers used by the Monte Carlo sample, i.e. the multidimensional unit cube. It is called the Primary Sample Space. Here the most valuable algorithms are Kelemen MLT [18], Multiplexed MLT [19] and RELT [20] and their further elaboration - MCPPM [21] and MBE [22]. The significant drawback of the MLT in the Path Space is that specific mutation strategies must be carefully designed for each illumination phenomenon and the perturbation probabilities $T(x \rightarrow y), T(x \leftarrow y)$ for the Metropolis rule which is not trivial. The hybrid method RJMLT [23] tries to overtake drawbacks of the methods working in the world Path Space or in the Primary Sample Space. It is able to work simultaneously in several spaces.

There are also methods which are based on the selection of sample population (PMC [24] and ERPT [25]). These methods keep information about samples in time and reuse the best samples as a starting point for mutations (small steps). 


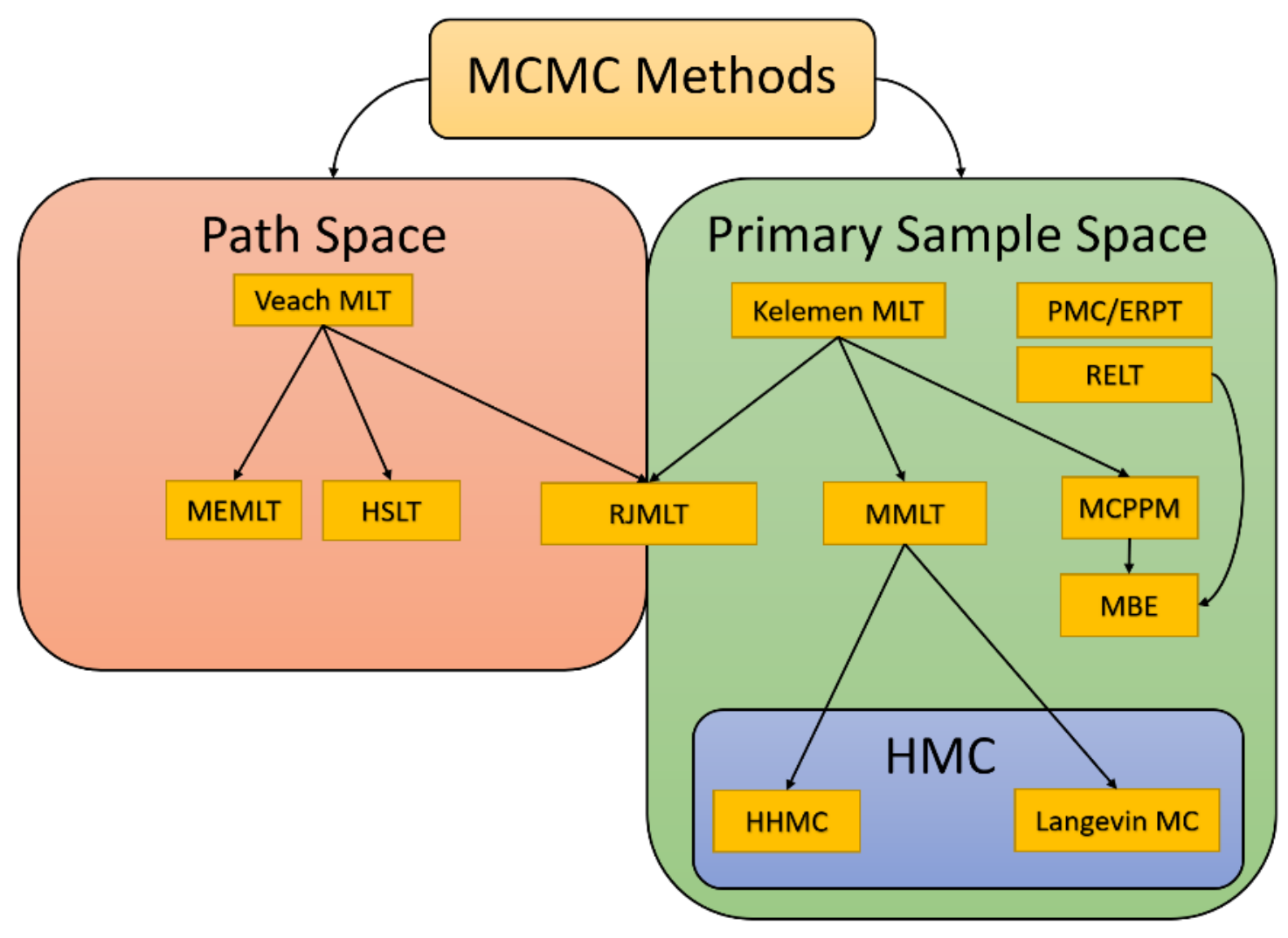

Figure 1: Classification for existing MCMC methods.

The new expanding class of methods is the hybrid Monte Carlo method [26]. These methods generate samples using trajectories of a dynamical system: Hamiltonian mechanics (HMC, Hamiltonian Monte Carlo) [27] or the Brownian motion in viscous medium based on the Langevin equation (LMC, Langevin Monte Carlo). There are just a few implementations of Hybrid Monte Carlo methods for rendering currently: HHMC [28], DRMLT (which is simplified version of HHMC) [29] and Langevin MC [30]. The ideas of the methods are described in details in [2]. The only note we should make here is that the light transport methods based on the hybrid Monte Carlo is a promising direction of research. These methods are universal, have a solid mathematical justification; they have better convergence when the integration space dimension grows [27]. This is due to the anisotropic transition proposal which is based on derivatives. The HMC significantly outperforms the MCMC because every "thin" region of the space gets even thinner as the dimension grows and the isotropic transition proposal in the MCMC has less chance to remain in the region of the function with high contribution.

\section{Classification \#2: Path Tracing vs Photon Mapping}

In addition to the classification into OMC and MCMC at least one more independent classification is possible:

1. methods operating in terms of luminance (like classic Monte Carlo Path Tracing);

2. methods operating with elements of a finite size in terms of the flux (like Photon Mapping).

This classification is possible both for the methods based on the OMC and for those based on the MCMC. However the methods working in terms of flux are mainly used within the OMC. Therefore, having both OMC/MCMC and Rays/Photons classification types we can position algorithms on a 2D map (Figure 2). 


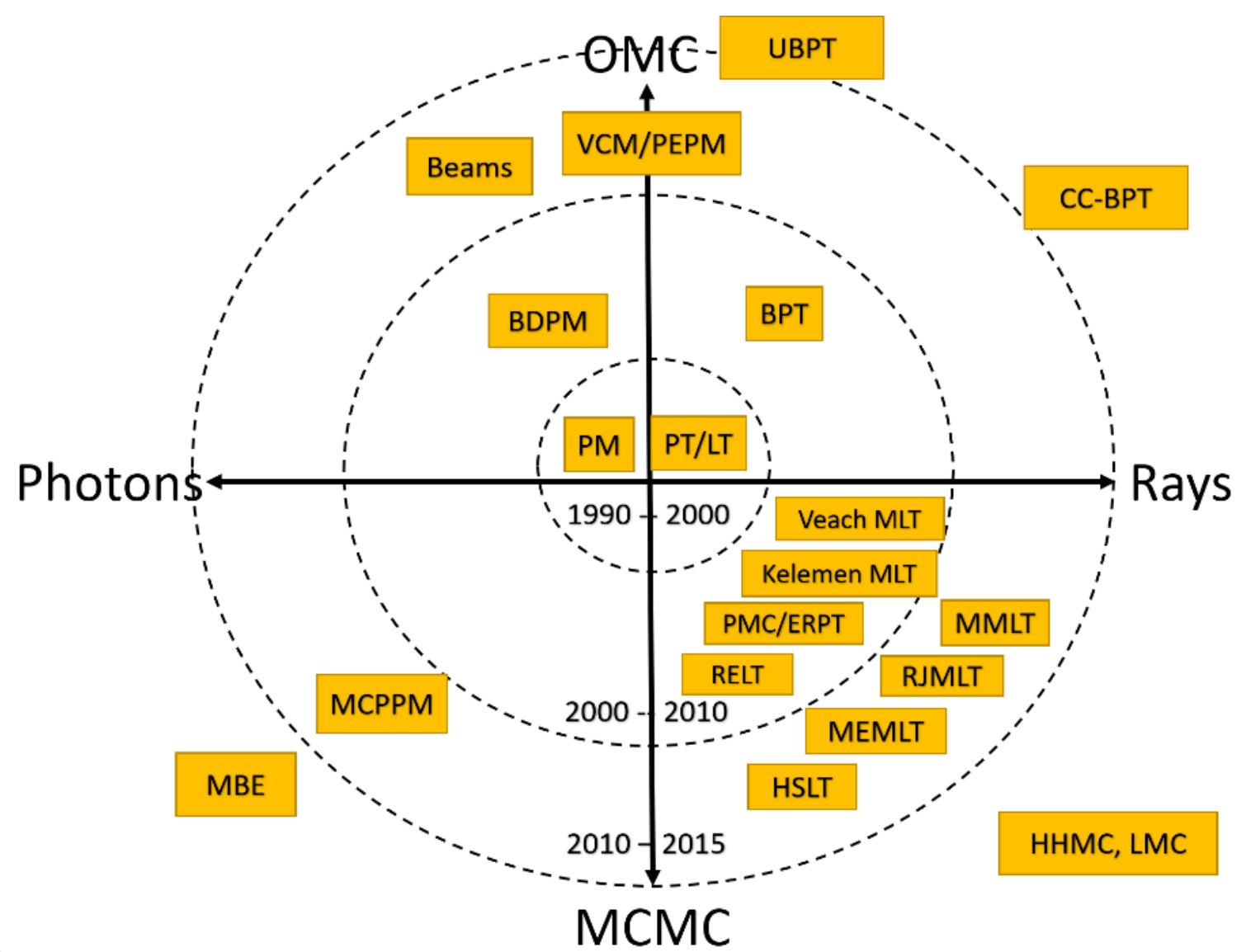

Figure 2: 2D view of light transport classification algorithms. Newer works are located further from the center of the image. At the top half of image OMC methods are presented. At the bottom half of image MCMC methods are shown. At the right part of the image classical Monte Carlo (Path Tracing) methods are presented. At the left part of the image density estimation (photon mapping) based methods are shown.

\subsection{Photon mapping}

Photon mapping (PM) uses biased estimate of the integral (in the standard definition of photon maps) rather than the unbiased estimate [31] and this biased estimate is consistent for progressive algorithms such as SPPM [32]. In practice this feature of the algorithm gives an approximate solution faster than for unbiased methods. However precise solution needs more time because simulation converges slower than for ray-based BPT method [33] which happens due to reducing the gathering radius in progressive methods. On the other hand the photon mapping is simpler than BPT and is able to calculate the illuminance for complex light paths with caustics. The caustic visualization problem is difficult for raybased methods and CC-BPT [12] was elaborated for this.

\subsection{Combining BPT and Photon Mapping}

The Final Gathering method gathers photons after the first non-specular bounce of ray during the backward tracing (Figure 3, FG). The photon maps are used in the final gathering as approximations of the third reflection and in this way they work very well for many scenes because the photon maps can quickly produce an approximate solution.

The Bidirectional Photon Mapping (BDPM) uses MIS to combine results for different bounces of Final Gathering [34] (Fig. 3, on the right). This improves robustness but reduces average speed due to expensive gathering operation happens on each bounce. Note that despite its name the BDPM is not a combination of the classical [31] and backward photon maps [35] because only the geometric problem 
of finding the closest photons is inverted in backward photon mapping while the computations of the integral and sampling do not change.

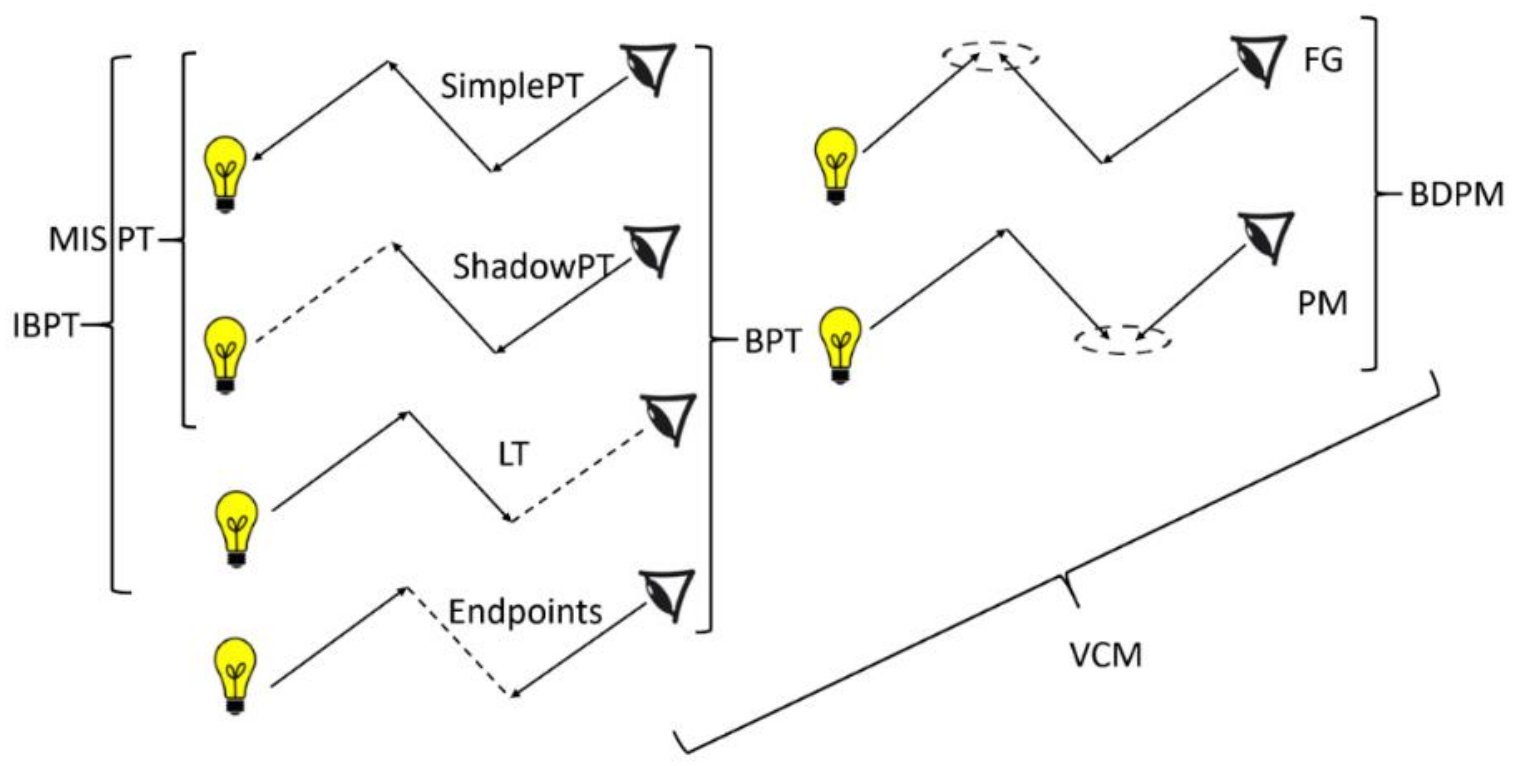

Figure 3: Sampling strategies and OMC methods which they yield in a combination

The Vertex Connection Merging (VCM) occupies true intermediate position between the first (BPT) and second (PM) classes because it integrates the photon maps in the BPT on the basis of MIS. In fact there are several such methods: VCM [36], PEPM [37], Beams and UBPT [38]. The MIS in these methods does not solve the problem of costly gathering operation because it works a posteriori, i.e. after the gathering procedure has been already performed. Figure 3 demonstrates how VCM is constructed from different strategies.

Currently the top of the development of MIS-based methods is CMIS [39] that allows one to extend the MIS to a continuum of sample generation strategies usually represented by a set of parametric functions. This improves convergence in many cases. However the problem of the optimal choice of strategies is not solved in the CMIS. This problem is solved in the Multiplexed Metropolis Light Transport (MMLT) algorithm only.

\section{Classification \#3: Generations of Light Transport algorithms}

In this section we group methods in conventional "generations" (Figure 4) by their efficiency for high precision simulation (for long calculations), general mathematical properties and restrictions. Thus, each generation has significantly different robustness for hard sampling problems and has different set of restrictions.

The First generations of methods is naive Monte-Carlo implementation: basic Path Tracing [4] and Photon Mapping [31] methods. The efficiency of these methods is extremely low (may depends, for example, from light source size or material properties) and often they can only be used for demonstration and studying purposes.

When Importance Sampling is applied we say Second generation of methods is used. An early versions of production software use so called "distributed/stochastic" ray tracing, Path Tracing and Photon Mapping. This generation of methods can already be used in practice but it is not robust even for calculation of direct illumination. As a result, many early renderers use tricks to suppress or clamp fireflies and due to that calculate light incorrectly. For example, "Simple PT" and "Shadow PT" (Figure 3) are of the second generation. They use two different sampling strategies: the Simple PT uses the implicit (material) sampling strategy and the Shadow PT uses the explicit one by issuing rays directly to the light source. 


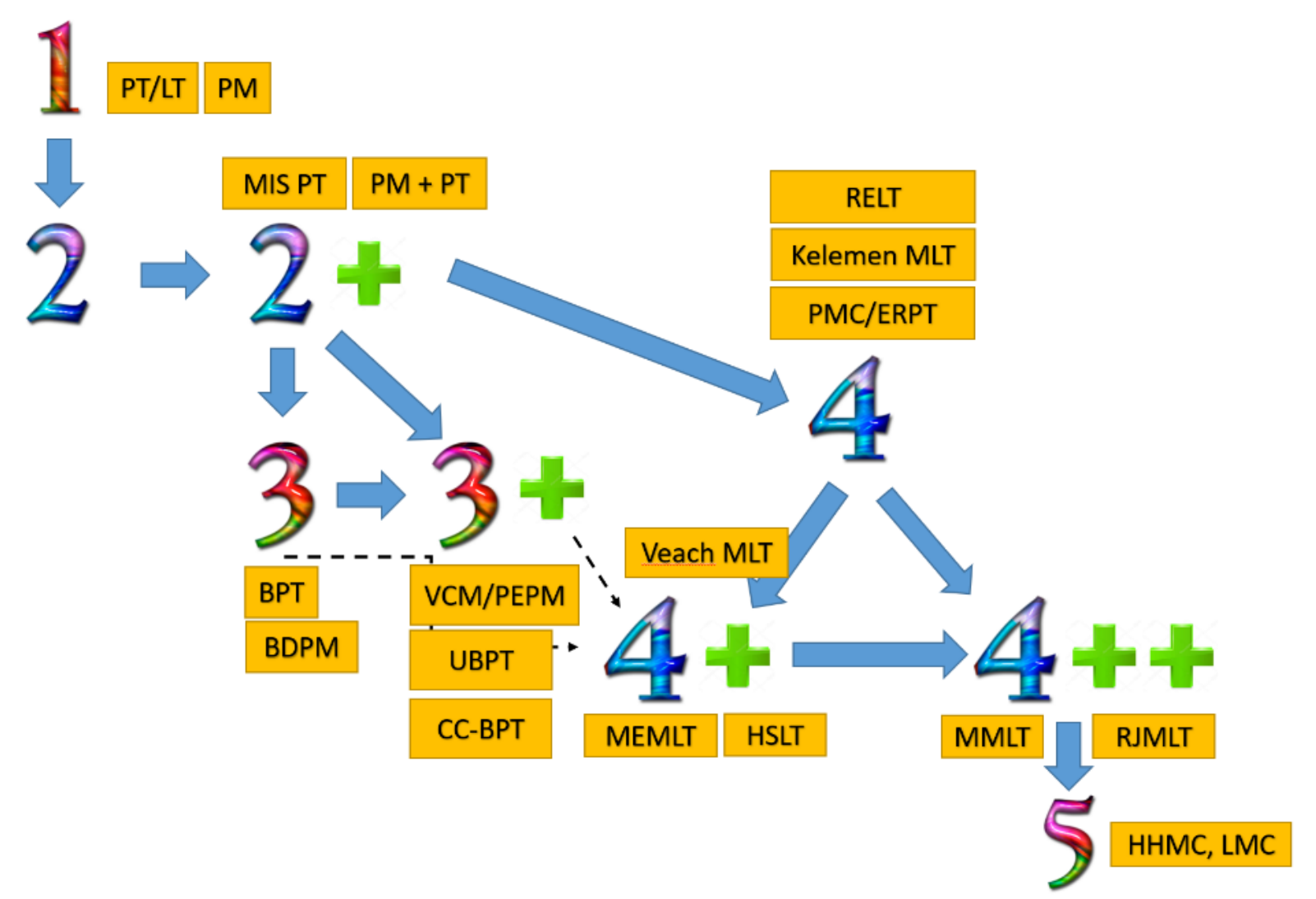

Figure 4: Generations of Monte Carlo Light Transport simulation methods. The numbers represent generations of methods, and the arrows between the numbers indicate that one generation inherits both the advantages and limitations of all previous generations. When generation number changes we move in bottom direction. In this way we show that generation number change means significant changes for mathematical foundations and framework used by methods of target generation.

Adding the Multiple Importance Sampling (MIS) in Path Tracing yields "Second Plus" (2+) Generation (MIS PT) which is robust for direct illumination. This is standard method for most of existing rendering systems today and can be used in many applications in practice. Here we already have serious complication for process of system expansion by new materials and light sources: the implementation of the material sampling (implicit strategies) and light sampling (explicit strategies) has to correctly calculate the Probability Density Function (PDF) for each sample. As a result, only highly specialized experts are able to elaborate the rendering system [5].

Adding photon mapping to MIS PT (this is done pretty often in practice) allows to efficiently evaluate several hard sampling phenomena like, for example, caustics. But such implementation is often accompanied by a lot of special cases and tricks and sometimes the rendering system works neither efficient, no correct. We attribute these methods to "Second Plus-Plus" (2++) Generation. Final Gathering [40] and its derivatives [41, 42] can be assigned here.

The Third generation of methods arises when MIS is applied to bidirectional methods on several light bounces. This is BPT [1] in Path Tracing world and BDPM [34] in the Photon Mapping world and all their derivatives methods like IBPT [6] and PCBPT [7]. In practice these methods are an order of magnitude more complicated for implementation than MIS PT because many different combinations of sampling strategies should be tested together to verify correctness of implementation for arbitrary scene. More significant problem here is that bidirectional methods impose a symmetry constraint on the material, geometry and light source models. In fact this is a fairly restrictive requirement as it was said above.

The "Third Plus" $(3+)$ generation is constructed by joining several methods of third generation together via Multiple Importance Sampling (MIS). These are VCM [36], PEPM [37], UBPT [38], CCBPT [12] and CMIS [39]. In fact, the algorithms of this generation are significantly more complicated for implementation than algorithms of the third generation ("... so much so that the authors also 
released a technical report and source code explaining how to implement the VCM algorithm..." [43]). The reason of complexity is a huge number of possible combinations of strategies in Multiple Importance Sampling.

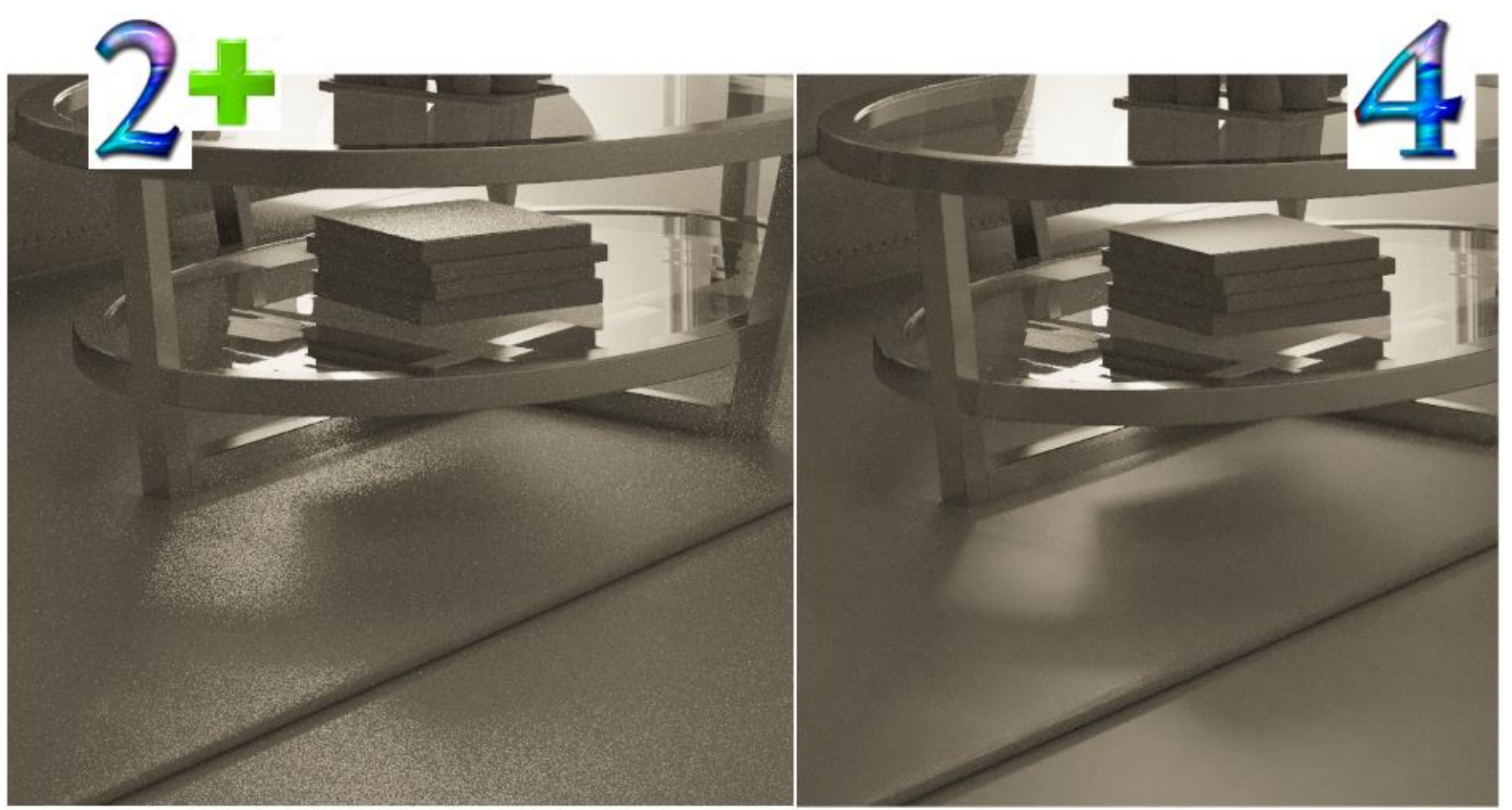

Figure 5: Comparison of 2+ (MIS PT) and 4-th generation (Kelemen MLT) methods with equal rendering time on scene with hard sampling lighting; our experiment.

Generations 3 and $3+$ are the latest generations where the methods based the Ordinary Monte Carlo are used. New methods arise but they use more and more complicated sampling strategies, strong restrictions (like differential geometry framework in CC-BPT) and a lot of heuristics. The problem here is that in integration space the dimension and complexity grows with the growing requirements to simulation system: layered materials, volume and sub-surface scattering [44], spectral calculations, light polarization, wave modeling [45] are good examples. We would like to simulate more and more complex light transport, do this precise and fast. That is why we need methods which are robust in general for multi-dimensional space where heuristic approach will fail sooner or later.

Therefore, the Fourth generation of methods is based on Markov Chain Monte Carlo and is constructed on top of unidirectional methods (Figure 5): Kelemen MLT [16], PMC [24, 25], RELT [20]. We believe this is a great point for practical implementation to stop because the only restriction of fourth generation methods (except for PDFs which we already have in $2+$ generation) is that direct light should be simulated in a separate pass with basic OMC method (for example, MIS PT). It is also quite easy to advance existing Path Tracer from MIS PT (2+) to Kelemen MLT (4). We only should change the process of random numbers generation and change the color accumulation algorithm. These methods also work well on GPU [46].

Consequently, the "Fourth Plus" (4+) generation uses Markov Chain Monte Carlo and constructed on top of bidirectional methods with different types of Multiple Importance Sampling. These are Veach MLT [13], MEMLT [16], HSLT [17], MCPPM [21, 47] and MBE [22]. We don't want to say all these methods have the same efficiency in practice. But all of them use MIS in a suboptimal way in comparison to further generations.

The "Fourth Plus-Plus" (4++) generation uses MIS in such a way that selection of sampling strategy is done by Markov Chain itself in statistically optimal way. So strategies with low contribution are selected rarely and high-contribution strategies selected often. This is also happens with shadows accounting. These are MMLT [19], RJMLT [23] and their derivatives. The MMLT algorithms is also simpler for implementation than any of the 4+ generation methods discussed above. Therefore, in our opinion MMLT is one of the best candidates for practical implementation of advanced light transport algorithm (Figure 6). Nevertheless, it is still much more complicated and restricted method than Kelemen MLT from the fourth generation and its GPU implementation has problems [48, 49]. 


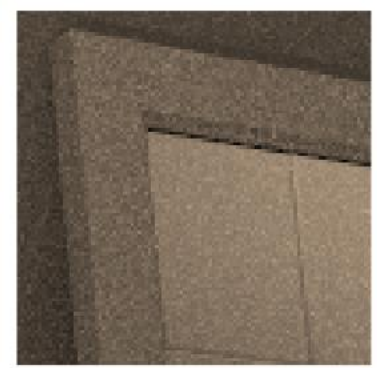

PT

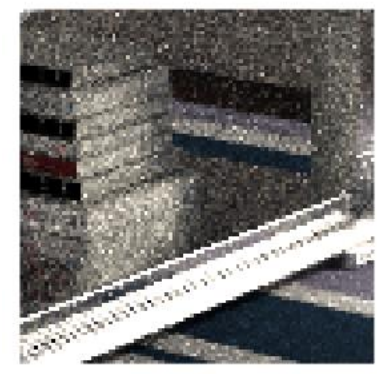

PT

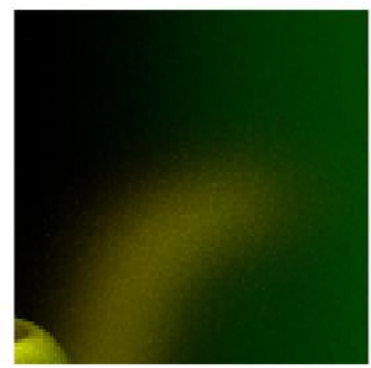

PT

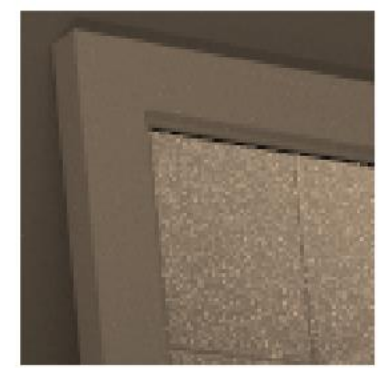

IBPT

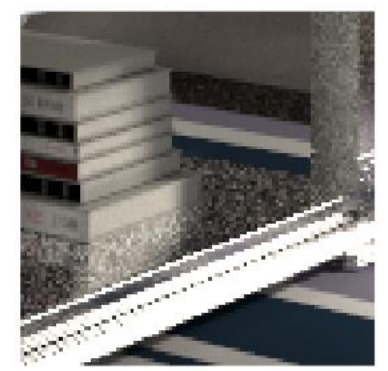

IBPT

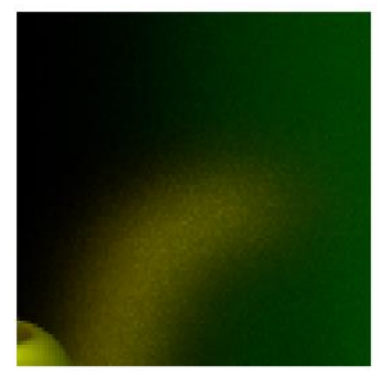

IBPT

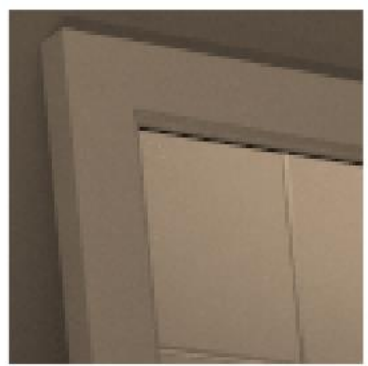

MMLT

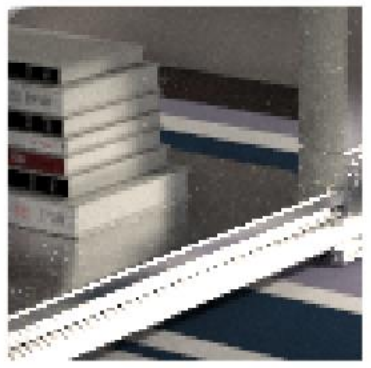

MMLT

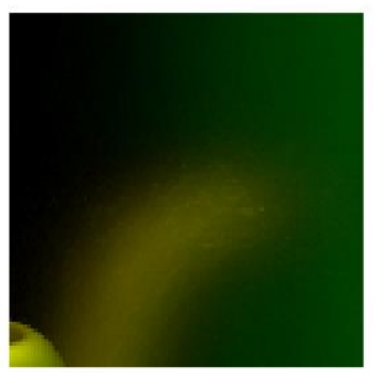

MMLT

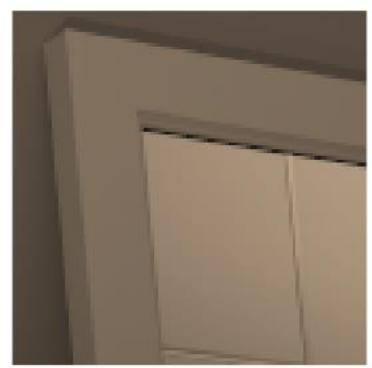

Reference

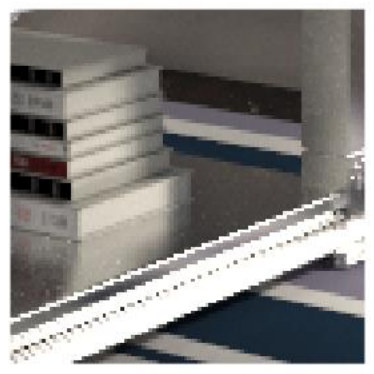

Reference

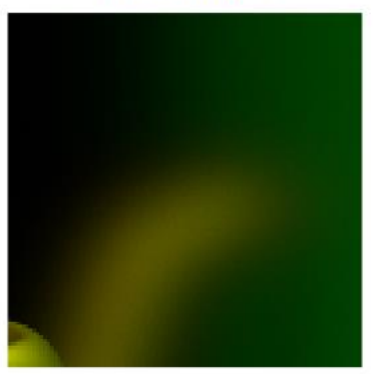

Reference

Figure 6: Comparison of MIS PT (2+), IBPT (3) and MMLT (4++) methods from left to right.

Finally, the Fifth generation methods are based on Hamiltonian and Langevin Monte Carlo. Light transport methods based on the hybrid Monte Carlo is a promising direction of research. These methods are universal, have a solid mathematical justification. They have better convergence when the integration space dimension grows. The main difficulty which prevents applying HMC and LMC methods in practice for today is requirement to apply automatic differentiation to contribution function. This is difficult and slow in general. Therefore existing implementations work on a restricted set of materials and light models, uses approximations and complex data structures.

\section{The guide}

Finally, we present a short practical guide for method selection (Figure 7).

It can be seen that we include quite old methods in the scheme. For example, the most recent work dates from 2014 year (MMLT). The reasons for this are:

1. We include here only basic methods. They are the mandatory checkpoints for any other advanced methods. Therefore, one should firstly implement one (or several) of these methods if plan to work with more advanced methods. For example, RELT may be constructed on top of Kelemen MLT; RJMLT and HHMC/LMC are all constructed on top of MMLT framework.

2. We include here only those methods which:

- $\quad$ have a few restrictions for used material, geometry and light sources models;

- $\quad$ have low complexity and thus are suitable for practical implementation in the software intended to be supported and elaborated by different people for many years. 


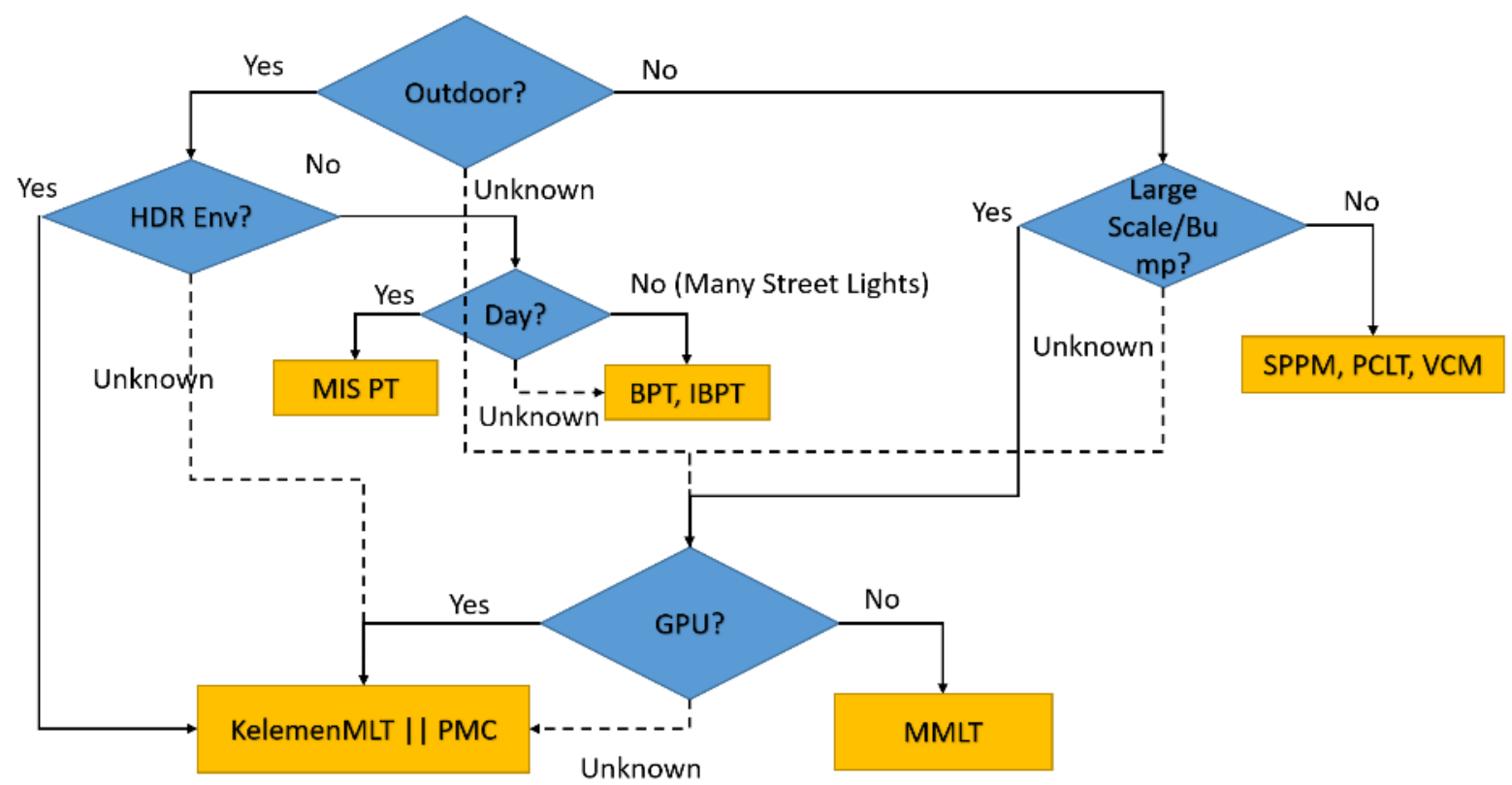

Figure 7: Block scheme for method selection. We show the most practical approaches from our previous survey [2].

\section{Conclusions}

Despite the significant progress in light transport simulations methods, there are just a few of them which are suitable for practical implementation. One of the biggest challenges is the uncontrolled growth of complexity for method implementation with the growing features and scene complexity. Another big problem is growing restrictions which makes newer methods impractical. It is easy to make an efficient light transport implementation within a restricted condition. For example, the SPPM computes caustics quite fast in scenes with limited space and simple materials.

However, it is extremely difficult to develop a general implementation which would be robust for all types of scenes and lighting phenomena. The Markov Chain methods are quire robust and efficient but even they have a flaw (at least they cannot calculate direct lighting). We also do not touch the inverse or differentiable rendering [50] which has a bunch of new problems.

Therefore, we believe that new approach to construction of render system should be developed. We do not really need one big light integration system. It is more preferable to use many small ones which can solve different problems within different scene restrictions efficiently. But on this way we have to develop a mechanism which ensures the compatibility and smooth transition between all these small renderers. This is the good field for future research.

\section{References}

[1] E. Veach, Robust Monte Carlo methods for light transport simulation, Ph.D. Dissertation, Stanford, Calif., Stanford University, 1998.

[2] V. A. Frolov, A. G. Voloboy, S. V. Ershov, V. A. Galaktionov, Light transport in realistic rendering: state-of-the-art simulation methods, Programming and Computer Software 47(4) (2021) 298-326. doi:10.1134/S0361768821040034.

[3] M. Sik, J. Krivanek, Survey of Markov chain Monte Carlo methods in light transport simulation, IEEE Trans.Visual. Comput. Graph. 26(4) (2018) 1821-1840. doi:10.1109/TVCG.2018.2880455

[4] J. T. Kajiya, The rendering equation, in: Proceedings of the 13th annual conference on Computer Graphics and Interactive Techniques (SIGGRAPH 86), 1986, pp. 143-150. 
[5] S. V. Ershov, A. G. Voloboy, Calculation of MIS weights for bidirectional path tracing with photon maps in presence of direct illumination, Math. Montisnigri 48 (2020) 86-102. doi:10.20948/mathmontis-2020-48-8

[6] D. Bogolepov, D. Ulyanov, GPU-optimized bidirectional path tracing, in: Proceedings of Conf. on Computer Graphics, Visualization and Computer Vision, 2013, pp. 1-15.

[7] S. Popov, R. Ramamoorthi, F. Durand, G. Drettakis, Probabilistic Connections for Bidirectional Path Tracing, Computer Graphics Forum 34(4) (2015) 75-86. doi:10.1111/cgf.12680.

[8] V. Schussler, E. Heitz, J. Hanika, C. Dachsbacher, Microfacet-based normal mapping for robust Monte Carlo path tracing, ACM Trans. Graph. 36(6) (2017) 205:1-205:12. doi: $10.1145 / 3130800.3130806$.

[9] S. H. Baek et al., Simultaneous acquisition of polarimetric SVBRDF and normals, ACM Trans. Graph. 37(6) (2018) 268:1-268:15. doi:10.1145/3272127.3275018

[10] C. Bar et al., A Monte Carlo framework for rendering speckle statistics in scattering media, ACM Trans. Graph. 38(4) (2019) 39:1-39:22. doi:10.1145/3306346.3322950

[11] A. Zhdanov, D. Zhdanov, V. Sokolov, I. Potemin, S. Ershov, V. Galaktionov, Problems of the realistic image synthesis in media with a gradient index of refraction, in: Proc. of SPIE 11548, Optical Design and Testing X., 2020, 115480W.

[12] S. Speierer, C. Hery, R. Villemin, W. Jako, Caustic connection strategies for bidirectional path tracing, Pixar Technical Memo, 2018, vol. 18-01.

[13] E. Veach, L. J. Guibas, Metropolis Light Transport, in: Proc. of the 24th Annual Conference on Computer Graphics and Interactive Techniques SIGGRAPH'97, 1997, pp. 65-76.

[14] M. Ashikhmin, S. Premoze, P. Shirley, B. Smits, A Variance analysis of the Metropolis light transport algorithm, Comput. Graphics (2001) 287-294.

[15] J. Kiivanek, I. Georgiev, A. S. Kaplanyan, J. Canada, Recent advances in light transport simulation: Theory and practice, ACM SIGGRAPH Courses, 2013.

[16] J. Wenzel, Light Transport on Path-Space Manifolds, Ph.D. Dissertation, Cornell University, 2013.

[17] A. S. Kaplanyan, J. Hanika, C. Dachsbacher, The natural-constraint representation of the path space for efficient light transport simulation, ACM Trans. Graph. 33(4) (2014) 102:1-102:13. doi:10.1145/2601097.2601108

[18] C. Kelemen, L. Szirmay-Kalos, G. Antal, F. Csonka, A simple and robust mutation strategy for the Metropolis light transport algorithm, in: G. Drettakis, H.-P. Seidel (ed.), Proc. of the EUROGRAPHICS, 2002, 21(3).

[19] T. Hachisuka, A. S. Kaplanyan, C. Dachsbache, Multiplexed Metropolis Light Transport, ACM Trans. Graph. 33(4) (2014) 100:1-100:10. doi:10.1145/2601097.2601138

[20] S. Kitaoka, Y. Kitamura, F. Kishino, Replica exchange light transport, Comput. Graphics Forum 28(8) (2009) 2330-2342.

[21] J. Chen, B. Wang, J.-H. Yong, Improved stochastic progressive photon mapping with Metropolis sampling, in: Proc. of the Twenty Second Eurographics Conference on Rendering (EGSR '11), 2011, pp. 1205-1213.

[22] M. Sik, H. Otsu, T. Hachisuka, J. Krivanek, Robust light transport simulation via Metropolised bidirectional estimators, ACM Trans. Graph. 35(6) (2016) 245:1-245:12. doi:10.1145/2980179.2982411

[23] B. Bitterli, W. Jakob, J. Novak, W. Jarosz, Reversible jump Metropolis light transport using inverse mappings, ACM Trans. Graph. 37(1) (2018). doi:10.1145/3132704

[24] Y. Lai, S. H. Fan, S. Chenney, C. Dyer, Photorealistic image rendering with population MonteCarlo energy redistribution, in: Proc. of the 18th Eurographics Conference on Rendering Techniques, 2007.

[25] D. Cline, J. Talbot, P. Egbert, Energy redistribution path tracing, ACM Trans. Graph. 24(3) (2005) $1186-1195$.

[26] S. Duane, A. D. Kennedy, B. J. Pendleton, D. Roweth, Hybrid Monte Carlo, Phys. Lett. 195(2) (1987) 216-222.

[27] S. Brooks, A. Gelman, G. Jones, X. Meng, MCMC using Hamiltonian dynamics, in: Chapman (ed.), Handbook of Markov Chain Monte Carlo, 2011, pp. 113-163. 
[28] T. Li, J. Lehtinen, R. Ramamoorthi, W. Jakob, F. Durand, Anisotropic Gaussian mutations for Metropolis light transport through Hessian-Hamiltonian dynamics, ACM Trans. Graph. 34(6) (2015) 209:1-209:13. doi:10.1145/2816795.2818084

[29] D. Rioux-Lavoie et al., Delayed rejection Metropolis light transport, ACM Trans. Graph. 39(3) (2020) 26:1-26:14. doi:10.1145/3388538

[30] F. Luan, S. Zhao, K. Bala, I. Gkioulekas, Langevin Monte Carlo rendering with gradient-based adaptation, ACM Trans. Graph. 39(4) (2020) 140:1-140:16. doi:10.1145/3386569.3392382

[31] H. W. Jensen, Global illumination using photon maps, in: Proceedings of Eurographics Workshop on Rendering techniques, Vienna: Springer, 1996, pp. 21-30.

[32] T. Hachisuka, H. W. Jensen, Stochastic progressive photon mapping, ACM SIGGRAPH Asia papers, 2009, pp. 1-8.

[33] A. S. Kaplanyan, C. Dachsbacher, Adaptive progressive photon mapping, ACM Trans. Graph. 32(2) (2013) 16:1-16:13. doi:10.1145/2451236.2451242

[34] J. Vorba, Bidirectional photon mapping, in: Proc. of the 15th Central European Seminar on Computer Graphics (CESCG) 2011, Prague, Charles University, 2011.

[35] V. Havran, R. Herzog, H.-P. Seidel, Fast final gathering via reverse photon mapping, EUROGRAPHICS 24(3) (2005).

[36] I. Georgiev, J. Krivanek, T. Davidovic, P. Slusallek, Light transport simulation with vertex connection and merging, ACM Transactions on Graphics 31(6) (2012) 192:1-192:10. doi:10.1145/2366145.2366211

[37] T. Hachisuka, J. Pantaleoni, H. W. Jensen, A path space extension for robust light transport simulation, ACM Trans. Graph 31(6) (2012) 191:1-191:10. doi:10.1145/2366145.2366210

[38] J. Krivanek, I. Georgiev, T. Hachisuka, P. Vevoda, M. Sik, D. Nowrouzezahrai, W. Jarosz, Unifying points, beams, and paths in volumetric light transport simulation, ACM Trans. Graphics (Proc. of SIGGRAPH) 33(4) (2014) 103:1-103:13. doi:10.1145/2601097.2601219

[39] R. West, I. Georgiev, A. Gruson, T. Hachisuka, Continuous multiple importance sampling, ACM Trans. Graph. 39(4) (2020) 136:1-136:12. doi:10.1145/3386569.3392436

[40] W. Jarosz, H. W. Jensen, C. Donner, Advanced global illumination using photon mapping, ACM SIGGRAPH 2008 classes (SIGGRAPH '08), New York, ACM, 2008, pp. 1-112.

[41] S. V. Ershov, D. D. Zhdanov, A. G. Voloboy, N. B. Deryabin, The method of quasi-specular elements to reduce stochastic noise in illumination simulation, Light Eng. 5 (2020) 39-47. doi:10.33383/2019-103.

[42] A. Zhdanov, D. Zhdanov, V. Galaktionov, Realistic image synthesis with hybrid photon maps, in: Proc. of SPIE, 2020, 115500G.

[43] J. Schutte, Vertex connection and merging, Rendering Equations Blog, 2018. URL: https://schuttejoe.github.io/post/vertexconnectionandmerging.

[44] J. Novák et al., Monte Carlo methods for volumetric light transport simulation, Comput. Graph. Forum, 37(2) (2018) 551-576. Doi:10.1111/cgf.13383

[45] S. Steinberg, L.-Q. Yan, A Generic Framework for Physical Light Transport . ACM Transactions on Graphics, Proceedings of SIGGRAPH 40(4) 2021 139:1-139:20. doi:10.1145/3450626.3459791

[46] V. A. Frolov, V. A. Galaktionov, Memory-compact Metropolis light transport on GPUs, Program. Comput. Software 43(3) (2017) 196-203.

[47] T. Hachisuka, H. W. Jensen, Robust adaptive photon tracing using photon path visibility, ACM Trans. Graph. 30(5) (2011) 114:1-114:11. doi:10.1145/2019627.2019633

[48] V. A. Frolov, Investigation of multiplexed Metropolis light transport on GPUs, Preprints of the Keldysh Institute of Applied Mathematics, 2018, 267. doi:10.20948/prepr-2018-267

[49] M. Schmidt, O. Lobachev, M. Guthe, Coherent Metropolis light transport on the GPU using speculative mutations, Journal WSCG 1 (2016).

[50] M. Nimier-David, D. Vicini, T. Zeltner, W. Jakob, Mitsuba2: a retargetable forward and inverse renderer, ACM Transactions on Graphics 38(6) (2019) 203:1-203:17. doi:10.1145/3355089.3356498. 